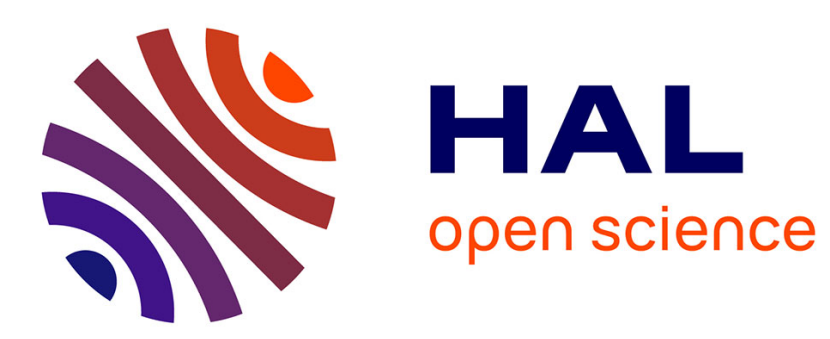

\title{
On-the-Fly Multi-Base Recoding for ECC Scalar Multiplication without Pre-Computations
}

\author{
Thomas Chabrier, Arnaud Tisserand
}

\section{To cite this version:}

Thomas Chabrier, Arnaud Tisserand. On-the-Fly Multi-Base Recoding for ECC Scalar Multiplication without Pre-Computations. ARITH - 21st IEEE International Symposium on Computer Arithmetic, Apr 2013, Austin, TX, United States. pp.219-228, 10.1109/ARITH.2013.17 . hal-00772613

\section{HAL Id: hal-00772613 https://hal.inria.fr/hal-00772613}

Submitted on 10 Jan 2013

HAL is a multi-disciplinary open access archive for the deposit and dissemination of scientific research documents, whether they are published or not. The documents may come from teaching and research institutions in France or abroad, or from public or private research centers.
L'archive ouverte pluridisciplinaire HAL, est destinée au dépôt et à la diffusion de documents scientifiques de niveau recherche, publiés ou non, émanant des établissements d'enseignement et de recherche français ou étrangers, des laboratoires publics ou privés. 


\title{
On-the-Fly Multi-Base Recoding for ECC Scalar Multiplication without Pre-Computations
}

\author{
Thomas Chabrier ${ }^{3,1}$ and Arnaud Tisserand ${ }^{2,1}$ \\ IRISA $^{1}$, CNRS $^{2}$, University Rennes $1^{3}$, INRIA Centre Rennes - Bretagne Atlantique \\ 6 rue Kerampont, CS 80518, 22305 Lannion cedex, FRANCE \\ arnaud.tisserandeirisa.fr
}

\begin{abstract}
Scalar recoding is popular to speed up ECC scalar multiplication: non-adjacent form, double-base number system, multi-base number system. But fast recoding methods require pre-computations: multiples of base point or off-line conversion. In this paper, we present a multi-base recoding method for ECC scalar multiplication based on i) a greedy algorithm starting least significant terms first, ii) cheap divisibility tests by multi-base elements and iii) fast exact divisions by multibase elements. Multi-base terms are obtained on-the-fly using a special recoding unit which operates in parallel to curve-level operations and at very high speed. This ensures that all recoding steps are performed fast enough to schedule the next curve-level operations without interruptions. The proposed method can be fully implemented in hardware without pre-computations. We report FPGA implementation details and very good performances compared to state-of-art results.
\end{abstract}

Index Terms - elliptic curve cryptography; scalar multiplication; DBNS; MBNS; divisibility test; exact division by constant;

\section{INTRODUCTION}

Scalar multiplication is the most time consuming operation in elliptic curve cryptography (ECC) protocols. It is denoted by $[k] P$ where $P$ is a curve point and $k$ a scalar. Basic scalar multiplication algorithm scans each bit of $k$ and performs some curve-level operations depending on the bit value. Scalar representation significantly impacts the number of point operations to be executed and overall computation time. Consequently scalar recoding methods are very popular: non-adjacent forms (NAF and $w \mathrm{NAF}$ ), double- or multi-base number systems (DBNS/MBNS), etc. Sec. II recalls these methods and basic ECC elements. Previous fast recoding methods require a precomputation step prior to scalar multiplication. For $w \mathrm{NAF}$, several multiples of $P$ have to be precomputed and stored. For DBNS/MBNS, the scalar must be recoded off-line.

Below we present a method and its FPGA implementation to recode on-the-fly the scalar using MBNS without precomputations. Our recoding is performed in parallel to curvelevel operations. It uses very cheap divisibility tests for each base element and an efficient implementation of exact division algorithms used for multiple-precision arithmetic. Exact division refers to division where the remainder is known to be zero. Due to paper length limit, we only deal here with curves defined over $\mathbb{F}_{p}$ but our method can be easily applied in $\mathbb{F}_{2^{m}}$ case. Sec. III and IV present respectively unsigned and signed versions of our method. Section V compares our results to state-of-art ones.

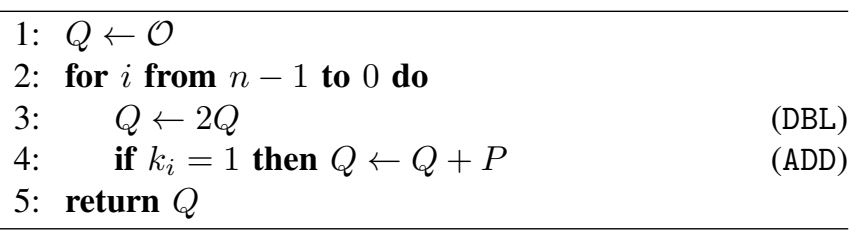

Fig. 1. Double-and-add algorithm for scalar multiplication $Q=[k] P$

\section{State-OF-ART in ECC Scalar Multiplication}

A brief introduction is presented below. The reader is referred to [1], [2] for further details. An elliptic curve $E$ over the prime field $\mathbb{F}_{p}$, of large characteristic, can be defined by the simplified Weierstrass equation $y^{2}=x^{3}+a x+b$ with curve parameters $a, b \in \mathbb{F}_{p}$ and $4 a^{3}+27 b^{2} \neq 0$. The rational points on the curve and a special point, called point at infinity denoted by $\mathcal{O}$, form an abelian group (denoted additively where $\mathcal{O}$ acts as the identity) on top of which the cryptosystem works. Given points $P, Q$ on the curve, curve-level operations are defined: point addition $P+Q$ where $P \neq \pm Q$ (denoted by ADD) and point doubling $[2] P=P+P$ (DBL). Scalar multiplication $[k] P$ is defined by $[k] P=P+P+\cdots+P$ with $k-1$ additions. The scalar $k$ is $\left(k_{n-1} k_{n-2} \ldots k_{1} k_{0}\right)_{2}$ with $n$ in the range $160-520$ bits for typical cryptographic sizes.

Each operation at curve-level involves a sequence of operations at field-level (multiplication: $\mathrm{M}$, square: S, inversion: I). Curve points can be represented using affine coordinates $(\mathcal{A})$ : $(x, y)$. In that case, $\mathrm{ADD}$ and $\mathrm{DBL}$ operations require expensive field inversions (in $\mathbb{F}_{p}$ one inversion is about 15 to 30 multiplications). Hence most efficient implementations use projective coordinates. In this paper, we use Jacobian coordinates $(\mathcal{J})$ as a popular class of projective coordinates where $(X: Y: Z)$ corresponds to the affine point $\left(X / Z^{2}, Y / Z^{3}\right)$ for $Z \neq 0$.

\section{A. Basic Scalar Multiplication Methods}

Basic scalar multiplication algorithm, called double-and$a d d$, is presented on Fig. 1. Its average computation cost is $0.5 n \mathrm{ADD}+n \mathrm{DBL}(0.5 n$ ones in $k$ for security requirement).

Point addition at line 4 always uses the same point $P$. Then $P$ can be kept in affine coordinates and used by mixed addition $\operatorname{mADD}(\mathcal{J}+\mathcal{A} \rightarrow \mathcal{J})$ in order to speed up the computation and reduce the $P$ coordinates storage (see Sec. V-A for cost).

Point subtraction (SUB) is as efficient as point addition $(\mathcal{A}$ : $-P=(x,-y)$ and $\mathcal{J}:-P=(X:-Y: Z)$ for curves 


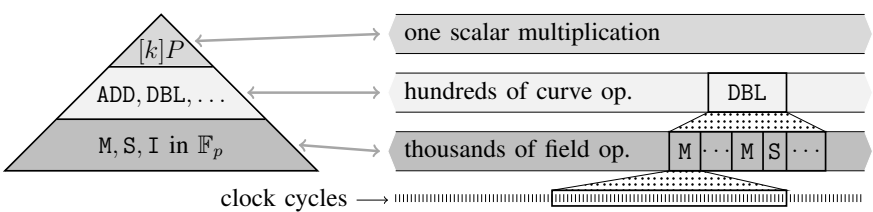

Fig. 2. Pyramid of operations in a scalar multiplication (arbitrary scale)

over $\mathbb{F}_{p}$ ). This motivates the use of signed digits such as NAF $\left(k_{i} \in\{0, \pm 1\}\right)$ where no two consecutive signed digits are non-zero [1, Sec. 3.3.1]. Scalar multiplication using NAF recoding is straightforward: replace line 4 in Fig. 1 by "if $k_{i} \neq 0$ then $Q \leftarrow Q \operatorname{sign}\left(k_{i}\right) P$ ". The average computation cost is $0.3 n \mathrm{ADD}+n \mathrm{DBL}$ (cost for SUB is the same than ADD).

Another optimization, called $w$ NAF, processes a window of $w$ digits of $k$ at a time. wNAF uses digits $k_{i} \in$ $\left\{0, \pm 1, \pm 3, \pm 5, \ldots, \pm 2^{w-1}-1\right\}$, and at most one of any $w$ consecutive digits is non-zero [1, Sec. 3.3.1]. Multiples $P_{j}=[j] P$ have to be pre-computed and stored for all $j \in\left\{3,5, \ldots, 2^{w-1}-1\right\}$. Scalar multiplication is done using ADD/SUB of pre-computed multiple $P_{j}$ (corresponding pseudocode is "if $k_{i} \neq 0$ then $Q \leftarrow Q \operatorname{sign}\left(k_{i}\right) P_{\mid k_{i}}$ "). The average computation cost is $n /(w+1) \mathrm{ADD}+n \mathrm{DBL}$ without the precomputation step. These pre-computations may be interesting if the same point $P$ is reused. In practice, $w \mathrm{NAF}$ is used with $w \leq 4$ for limited storage overhead.

Fig. 2 illustrates the typical number of operations required at each level. One $[k] P$ operation requires hundreds of curvelevel operations. Each curve operation (ADD, DBL) requires a sequence of 8-12 field-level operations. Finally, each field operation requires tens (for large operators) to hundreds (for small iterative operators) of clock cycles.

\section{B. Scalar Multiplication using Double-Base Number System}

DBNS was initially introduced in [3], used for modular exponentiation in [4], for signal processing in [5] and for ECC in [6], [7], [8], [9], [10] and [11] (which is very complete). In DBNS, number $x$ is represented by the sum of mixed powers of two co-prime integers $b_{1}$ and $b_{2}$, the two bases, typically $\left(b_{1}, b_{2}\right)=(2,3)$, such that $x=\sum_{i=1}^{n^{\prime}} x_{i} b_{1}^{u_{i}} b_{2}^{v_{i}}$ with $x_{i}= \pm 1$. Unsigned DBNS $\left(x_{i}=1\right)$ leads to larger terms number $n^{\prime}$.

For ECC computations in DBNS, a new curve-level operation has to be defined: point tripling [3] $P=P+P+P$ (denoted TPL). It is faster than ADD (see Sec. V-A for cost). DBNS is a very sparse representation (number of terms $n^{\prime}$ is very small compared to number of bits $n$ in standard binary representation). Then, the number of point additions is reduced. In [7], a special type of DBNS recoding, called DBNS chain, is proposed with an Horner like factorization of DBLs and TPLs operations (under conditions $u_{1} \geq u_{2} \geq \ldots \geq u_{n^{\prime}}$ and $v_{1} \geq v_{2} \geq \ldots \geq v_{n^{\prime}}$ ) leading to higher improvement. We will report performances of some DBNS scalar multiplication algorithms from literature in Sec. V. There are DBNS scalar multiplication extensions using pre-computed multiples of $P$ leading to higher speed but with a higher storage cost [11].
DBNS helps to reduce the total computation time. But binary to DBNS conversion is performed off-line. Most of proposed conversions proceed most significant terms first by subtracting to $k$ a good/best approximation of $k$ by a term of form $2^{u} 3^{v}$ using huge tables or expensive computations. The authors from [10] claim that tree based approach conversion is too costly for hardware implementation of systems using integers in the cryptographic range (p. 437, Sec. 3). In [8], 10 to 72 points have to be pre-computed and stored (a better usage of silicon area should be a parallel architecture). In [12], an FPGA implementation of binary to DBNS conversion is proposed but only for very small operands ( $n \leq 20$ bits) in signal processing applications.

DBNS is a very redundant number system. In [13], this redundancy is used to randomly select the recoding as a counter-measure against some side-channel attacks (SCAs).

\section{Scalar Multiplication using Multi-Base Number System}

MBNS is a generalization of DBNS with more than two bases [14], [15], [16], [17], [18], [19] and [20]. A multi-base $\mathcal{B}$ is a tuple of $l$ co-prime integers $\left(b_{1}, b_{2}, \ldots, b_{l}\right)$. Number $x$ is represented as the sum of terms $x=\sum_{i=1}^{n^{\prime}}\left(x_{i} \prod_{j=1}^{l} b_{j}^{e_{j, i}}\right)$ with $x_{i}= \pm 1$. MBNS is a very sparse and redundant representation. In literature, proposed multi-bases are often $(2,3,5)$ and $(2,3,5,7)$.

For ECC scalar multiplication in MBNS, new curve-level operations have to be defined: point quintupling [5]P (QPL), point septupling $[7] P$ (SPL), point eleventupling [11]P (EPL), etc. These new operations are more efficient than equivalent sequences of ADD, DBL and TPL operations (see Sec. V-A for typical costs). MBNS scalar multiplication is similar to DBNS algorithms with more curve-level operations QPL, SPL, etc.

MBNS suffers from the same limitation as DBNS: the need for off-line conversion with huge tables and/or long pre-computations. In [14] and [17] conversion uses good approximations of $k$ using terms of form $\pm \prod_{j=1}^{l} b_{j}^{e_{j}}$ similarly to DBNS conversion. In [15] and [16] conversion uses an adaptation of $w$ NAF with detection of $b_{j}$ multiples into a limited window, but it requires pre-computations and additional storage. To our knowledge, [15] and [16] provide the best MBNS results but without hardware implementation details.

\section{Proposed Method}

Notations used in paper remainder are:

- $k=\left(k_{n-1} k_{n-2} \ldots k_{1} k_{0}\right)_{2}, k>1$, the $n$-bit scalar stored into $t$ words of $w$ bits with $w(t-1)<n \leq w t$ (i.e. last word may be 0-padded). $k^{(i)}$ the $i$ th word of $k$ starting from least significant for $0 \leq i<t$.

- $\mathcal{B}$ the multi-base with $l$ base elements (co-prime integers), $\mathcal{B}=\left(b_{1}, b_{2}, \ldots, b_{l}\right)$.

- predicate divisible $(x, \mathcal{B})$ returns true if $x$ is divisible by at least one base element in $\mathcal{B}$ (false for other cases).

- number $x$ represented as the sum of terms $x=$ $\sum_{i=1}^{n^{\prime}}\left(d_{i} \prod_{j=1}^{l} b_{j}^{e_{j, i}}\right)$ with $d_{i}= \pm 1$.

- $\operatorname{term}\left(d_{i}, e_{1, i}, e_{2, i}, \ldots, e_{l, i}\right)$ defined by $d_{i} \times \prod_{j=1}^{l} b_{j}^{e_{j, i}}$ in $\mathcal{B}$ (index $i$ may be omitted when context is clear). 


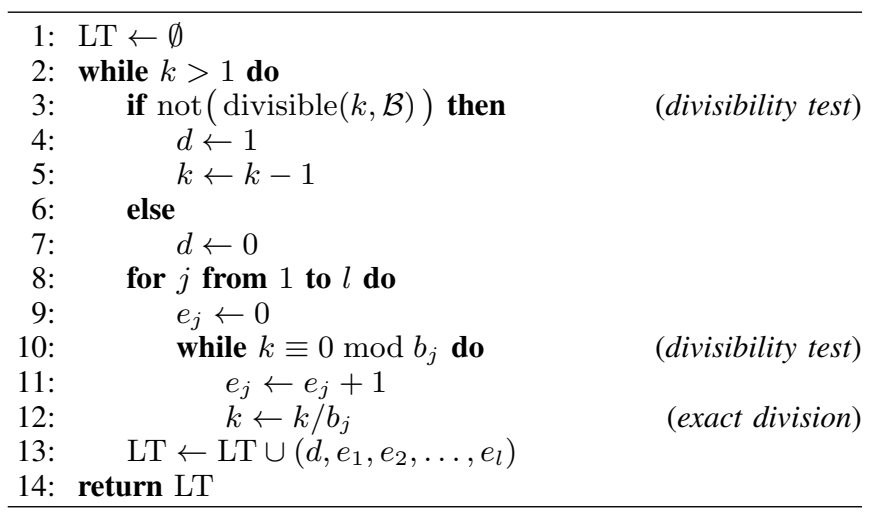

Fig. 3. Unsigned MBNS recoding algorithm

- $Q, P$ curve points and $Q=[k] P$ scalar multiplication.

Due to space limitation, we only present results for elliptic curves defined over $\mathbb{F}_{p}$, but it can be used for $\mathbb{F}_{2^{m}}$ (fine tuning is slightly different due to different cost ratios $\mathrm{I} / \mathrm{M}$ and $\mathrm{S} / \mathrm{M}$ ). In this section, we present a simple unsigned version $\left(d_{i}=1\right)$ for the sake of simplicity. Sec. IV details algorithms for signed representations $\left(d_{i}= \pm 1\right)$. Units described in this section can be directly used or slightly adapted for signed representation.

\section{A. Unsigned Algorithms}

Our MBNS unsigned recoding algorithm, see Fig. 3, is very simple. Divisibility of $k$ by $\mathcal{B}$ elements is tested. When $k$ is not divisible, 1 is subtracted to $k . b_{1}=2$ is selected for efficiency purpose (divisibility is ensured $50 \%$ of time). For lines $8-12$, the scalar $k$ is divided by all base elements $b_{j}$ in $\mathcal{B}$ as much as possible using cheap divisibility tests and exact divisions. This division step provides the term exponents $e_{1}, e_{2}, \ldots, e_{l}$. LT denotes the list of terms which stores the MBNS recoding of $k$, $\mathrm{LT}=\left(\left(d_{1}, e_{1,1}, e_{2,1}, \ldots, e_{l, 1}\right),\left(d_{2}, e_{1,2}, e_{2,2}, \ldots, e_{l, 2}\right), \ldots\right)$ with $d_{i} \in\{0,1\}$. Only the first term may have $d_{1}=0$ (if the initial $k$ is immediately divisible in $\mathcal{B}$ ). Divisibility tests at line 3 and 10 can be shared. The algorithm stops when $k \leq 1$ due to Horner form such as $2^{a} 3^{b} 5^{c}\left(1+2^{a^{\prime}} 3^{b^{\prime}} 5^{c^{\prime}}(1)\right)$.

Divisibility tests are detailed in Sec. III-B. There are implemented using $t+\varepsilon$ clock cycles ( $\varepsilon$ is a small constant) for all $b_{j} \neq 2$ and only one for $b_{j}=2^{s}$ with $s \leq w$. Once $k$ is divisible by $b_{j}$, we use fast exact division algorithms to perform $k \leftarrow k / b_{j}$ as detailed in Sec. III-C and with $t+\varepsilon^{\prime}$ clock cycles for all $b_{j} \neq 2$.

MBNS recoding algorithm in Fig. 3 works in a serial way: one multi-base term at a time and starting with the least significant one. Each term can be immediately used in the scalar multiplication algorithm in Fig. 4. This algorithm computes $Q=[k] P$ using LT and is a multi-base adaptation of the standard left-to-right scalar multiplication algorithm (see for instance [1, Sec. 3.3.1]). Operation $Q+d \times P$ at line 3 is NOP (no operation) or ADD since $d \in\{0,1\}$.

The combination of recoding (Fig. 3) and scalar multiplication (Fig. 4) algorithms allows to overlap recoding steps by curve-level operations. For instance, when divisibility by 3 is detected, exact division by 3 and TPL operations can

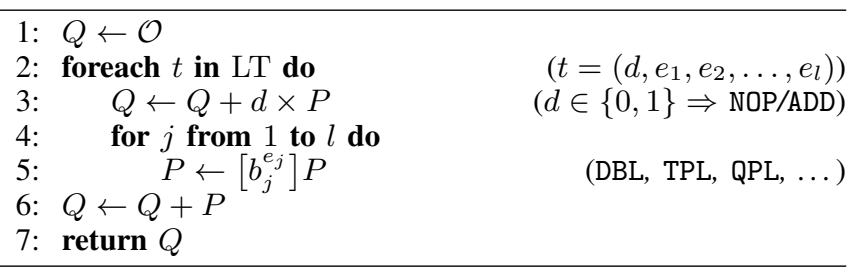

Fig. 4. MBNS scalar multiplication algorithm $Q=[k] P$

be launched in parallel. Our recoding algorithm produces a MBNS representation with a recursive factorization similar to Horner scheme. Fig. 10 illustrates a complete example. Unlike previous DBNS and MBNS recoding methods, ours can be fully embedded in hardware and operates on-the-fly. First, we do not need costly tables or computations such as the approximation of $k$ by multi-base terms. Second, as soon as a divisibility is detected, we can launch the corresponding curve-level operation.

As we start with least significant terms first, we cannot use mixed coordinates point addition (mADD). We are obliged to use standard point addition which is a little slower. Clearly our method is not competitive compared to the fastest stateof-art ones when costly off-line recoding is possible. But it provides the first full on-the-fly hardware implementation.

Overlapping recoding operations by curve-level operations is possible due to the very fast divisibility tests and exact divisions. For instance, with $n=160, w=12$ and $t=14$, divisibility tests by all $b_{j} \neq 2$ and exact division by one $b_{j} \neq 2$ respectively require $t+3=17$ and $t+4=18$ clock cycles. These small durations have to be compared to the duration of one DBL, TPL, QPL, etc. which are significantly slower.

There is a short latency at the very beginning (less than $0.01 \%$ of total $[k] P$ computation time for $n=160$ and even less for larger fields). The first curve-level operation is determined using the first divisibility test results. After $t+\varepsilon$ clock cycles there are two cases: i) $k$ is divisible by one multibase element $b_{j}$ then a DBL, TPL, QPL, etc. can be launched depending on which $b_{j}$ divides $k$, ii) $k$ is not divisible by $\mathcal{B}$ elements and an ADD can be launched.

In this work we do not study an analytical evaluation of the number of each type of curve-level operation. But we provide extensive experimental evaluations of both these types of operations and complete scalar multiplication duration.

Selection of $\mathcal{B}$ elements is required. Fig. 5 reports statistical $[k] P$ timings (in M) for 10000 random 160-bit values recoded using our unsigned MBNS algorithm for various multi-bases.

Scalar multiplication algorithm in Fig. 4 is not secure

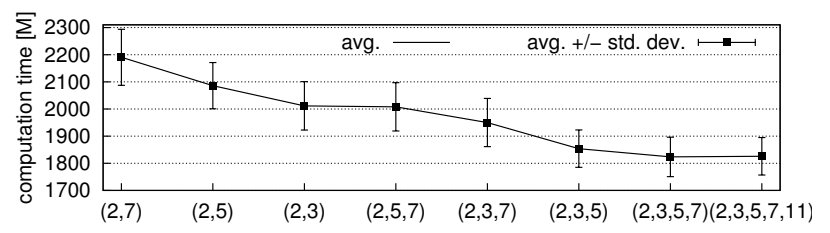

Fig. 5. Statistical timings of unsigned MBNS scalar multiplication 


\begin{tabular}{|c||cccccccccccc|}
\hline \multicolumn{1}{|c||}{} & \multicolumn{110}{c|}{$i$} \\
\cline { 2 - 12 }$b_{j}$ & 11 & 10 & 9 & 8 & 7 & 6 & 5 & 4 & 3 & 2 & 1 & 0 \\
\hline \hline 3 & 2 & 1 & 2 & 1 & 2 & 1 & 2 & 1 & 2 & 1 & $\mathbf{2}$ & $\mathbf{1}$ \\
5 & 3 & 4 & 2 & 1 & 3 & 4 & 2 & 1 & $\mathbf{3}$ & $\mathbf{4}$ & $\mathbf{2}$ & $\mathbf{1}$ \\
7 & 4 & 2 & 1 & 4 & 2 & 1 & 4 & 2 & 1 & $\mathbf{4}$ & $\mathbf{2}$ & $\mathbf{1}$ \\
\hline
\end{tabular}

TABLE I

PASCAL'S TAPES FOR DIVISIBILITY TESTS (VALUES ARE $2^{i} \bmod b_{j}$ )

against SCAs. Simple power analysis attacks can be used due to the different behavior of curve-level operations in lines 3 and 5. We will see in Sec. IV how signed MBNS recoding can be used as a protection against some attacks.

\section{B. Implementation of the Divisibility Tests}

At each recoding step, the scalar remainder has to be tested for divisibility by all $b_{j}$ for $1 \leq j \leq l$. Testing divisibility by $2^{s}$ with $s \leq w$ in a radix-2 representation is straightforward and implemented in a very small module of the recoding unit, see Sec. III-D. For $b_{j} \neq 2$, we use a very old method based on specific properties of the sum of argument digits modulo $b_{j}$. This method for divisibility test by $b_{j}$ in radix $r$ representation is reported in a Blaise Pascal's post-mortem publication [21] (in Latin, see [22] for comments in English). This method is often called Pascal's tape. We only provide details for $b_{j} \in$ $\{3,5,7\}$ as they lead to the most efficient $\mathcal{B}$. Tab. I reports the remainders $2^{i} \bmod b_{j}$. They form a periodic sequence.

Using Tab. I with Pascal's tape in case $b_{j}=3$, the periodic sequence is $(21)^{*}$, then one has:

$$
\begin{aligned}
k \bmod 3 & =\left(\ldots+2^{3} k_{3}+2^{2} k_{2}+2^{1} k_{1}+k_{0}\right) \bmod 3 \\
& =\left(\ldots+2 k_{3}+k_{2}+2 k_{1}+k_{0}\right) \bmod 3 \\
& =(\underbrace{\sum\left(2 k_{2 i+1}+k_{2 i}\right)}_{\alpha}) \bmod 3 .
\end{aligned}
$$

Computation of $\alpha$ requires the sum of many 2-bit words $(n \gg 100) . \alpha$ is a multi-bit integer, then it has to be recursively reduced using the same method. There is a trade-off between the size of the intermediate accumulators and the reduction completion. Architecture presented on Fig. 6 decomposes this large operation into partial sums accumulated and partial reduction on a limited number of bits for each word of the scalar. This is the purpose of the light block denoted " $\sum$ for $b_{j}=3$ " and connected register on Fig. 6 . Then $t$ cycles are required for the accumulation and partial reduction. The very last reduction steps and comparison to $b_{j}$ is denoted " $\mathcal{R}$ for $b_{j}$ " in the second type of light block. Clock, reset and enable signals are not represented on the figures.

For $b_{j}=7$, the sum uses 3-bit words with the sequence $(421)^{*}$. But for $b_{j}=5$, the sequence is $(3421)^{*}$ where digit 3 requires a specific treatment. A first solution uses $3=1+2$ and an unsigned sum with additional inputs. A second solution considers $3 \equiv-2 \bmod 5$ and a signed sum with less operands (but with sign extension). Architecture on Fig. 6 allows parallel divisibility test by several $b_{j}$ in only one computation.

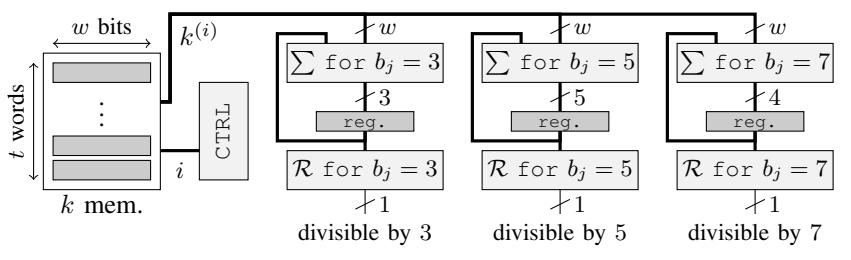

Fig. 6. Divisibility tests architecture

\begin{tabular}{|c||c|c|c|}
\hline$w$ & $\begin{array}{c}\text { area } \\
\text { slices (FF/LUT) }\end{array}$ & $\begin{array}{c}\text { freq. } \\
\text { MHz }\end{array}$ & $\begin{array}{c}\text { clock } \\
\text { cycles }\end{array}$ \\
\hline \hline 12 & $25(40 / 81)$ & 543 & $t+3$ \\
\hline 24 & $67(53 / 152)$ & 549 & $t+4$ \\
\hline
\end{tabular}

TABLE II

FPGA IMPLEMENTATION RESULTS FOR DIVISIBILITY TESTS

Parameter $w$ significantly impacts performances. The lengths of remainders sequences for $b_{j}=3,5,7$ are respectively $2,4,3$ (from Tab. I). To avoid complex decoding schemes, we use $w=\operatorname{lcm}(2,4,3)=12$ and $w=24$ (larger multiples of 12 slow down the recoding unit).

Hardware implementations reported below have been described in VHDL and implemented on a XC5VLX50T FPGA using ISE 12.4 from Xilinx with standard efforts for synthesis, place and route. We report numbers of clock cycles, best clock frequencies and numbers of occupied slices. We also report numbers of look-up tables (LUTs with 6 inputs in Virtex 5) and flip-flops (FFs) for area. A XC5VLX50T contains 7200 slices with 4 LUT and 4 flip-flops per slice. We use flip-flops for all storage elements. FPGA implementation results are reported in Tab. II for divisibility tests by $b_{j} \in\{3,5,7\}$ and $n=160$.

\section{Implementation of the Exact Division by $b_{j}$ Elements}

Here, exact division $k / b_{j}$ means that we know that dividend $k$ is divisible by divisor $b_{j}$ (using divisibility tests presented above). This significantly optimizes the division. [23] provides an efficient algorithm when the radix is prime or power of 2 . Division by $2^{s}$ is straightforward and is not considered in this section (a shifter is included in the recoding unit Sec. III-D).

We use a dedicated version of algorithm presented in [23] for $b_{j} \in\{3,5,7\}$ and optimized for hardware implementation. Our algorithm, presented in Fig. 7, operates in $t$ iterations in a word-serial way starting with least significant. Iteration number $i$ deals with $k^{(i)}$ the $i$ th word of $k$. The inverse of divisor $b_{j}$ modulo $2^{w}$ is a constant and always exists since multi-base elements are co-prime and include 2 .

The main differences for the 3 operations $\left(b_{j} \in\{3,5,7\}\right)$ are the multiplication by modular inverse on line 4 and comparisons to constants on line 7. All other elements are shared in the architecture (operators, control, registers).

Tab. III reports binary representations of modular inverses for exact division by 3,5,7. Multiplication $r \times\left(b_{j}^{-1} \bmod 2^{w}\right)$ at line 4 in Fig. 7 is implemented as a sequence of additions/subtractions and shifts using an in-house multiplication by constants algorithm [24]. Subtraction at line 3 is 


\begin{tabular}{|c||c|c|}
\hline$b_{j}$ & $b_{j}^{-1} \bmod 2^{12}, \gamma$ & $b_{j}^{-1} \bmod 2^{24}, \gamma$ \\
\hline \hline 3 & $(101010101011)_{2}, 3$ & $(101010101010101010101011)_{2}, 4$ \\
\hline 5 & $(110011001101)_{2}, 3$ & $(110011001100110011001101)_{2}, 4$ \\
\hline 7 & $(110110110111)_{2}, 3$ & $(110110110110110110110111)_{2}, 4$ \\
\hline
\end{tabular}

TABLE III

MODULAR INVERSES USED IN EXACT DIVISIONS

\begin{tabular}{|c||c|c|c|}
\hline$w$ & $\begin{array}{c}\text { area } \\
\text { slices (FF/LUT) }\end{array}$ & $\begin{array}{c}\text { freq. } \\
\text { MHz }\end{array}$ & $\begin{array}{c}\text { clock } \\
\text { cycles }\end{array}$ \\
\hline \hline 12 & $59(138 / 171)$ & 291 & $t+4$ \\
\hline 24 & $152(441 / 448)$ & 202 & $t+5$ \\
\hline
\end{tabular}

TABLE IV

FPGA IMPLEMENTATION RESULTS FOR EXACT DIVISION.

inserted in the sequence. Some adders in the 3 sequences are shared to reduce area. Tab. III reports $\gamma$ the number of additions/subtractions required to perform $r \times\left(b_{j}^{-1} \bmod 2^{w}\right)$.

The architecture of our exact division by $b_{j} \in\{3,5,7\}$ unit is presented on Fig. 8. At iteration $i$, word $k^{(i)}$, read (R port) from scalar memory, is added to $-c$ and used in the addition sequence (block denoted " $\times\left(b_{j} \bmod 2^{w}\right)$ " and " \pm seq.") corresponding to $b_{j}$. The correct value is selected by MUX1 and written in scalar memory (W port). We use an in-place version of the algorithm $k \leftarrow k / b_{j}$ to keep memory footprint as small as possible. Comparisons in loop lines 6-8 of algorithm Fig. 7 are unrolled and implemented as combinatorial logic (" $\mathrm{cmp}$. $b_{j}$ " block) for each $b_{j} \in\{3,5,7\}$. The correct value $c$ is selected by MUX2 and sent the addition sequences. The FPGA implementation results for our exact division unit are reported in Tab. IV for $n=160$. For $w=24$, speed decreases due to the complexity of the comparison blocks. For example, in case $b_{j}=7$, six comparisons are required.

\section{Unsigned Multiple-Base Recoding Unit}

The complete recoding unit architecture is presented on Fig. 9. The scalar memory stores $k$. The small subtraction block is in charge of line 5 in the recoding algorithm Fig. 3. The DTD-2 block is in charge of divisibility test (1-bit result) and exact division ( $w$-bit bus) by 2 or small powers of 2 ( $2^{s}$ with $s \leq w$, if $s>w$ several iterations are used). Divisibility test unit for other base elements is described in Sec. III-B (3-bit output) while the exact division unit is

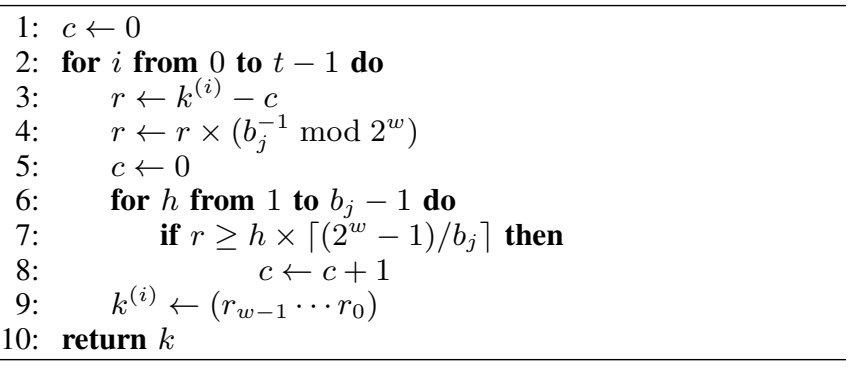

Fig. 7. Exact division algorithm for $k / b_{j}$

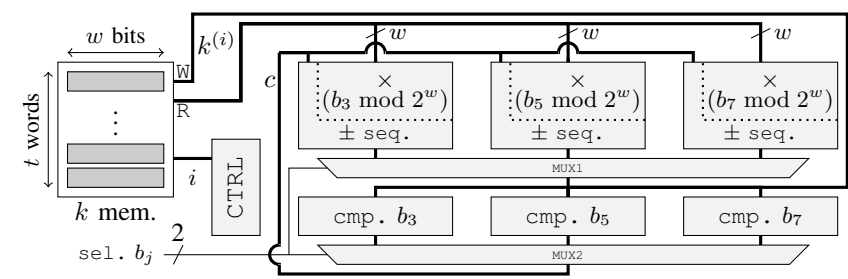

Fig. 8. Exact division unit architecture

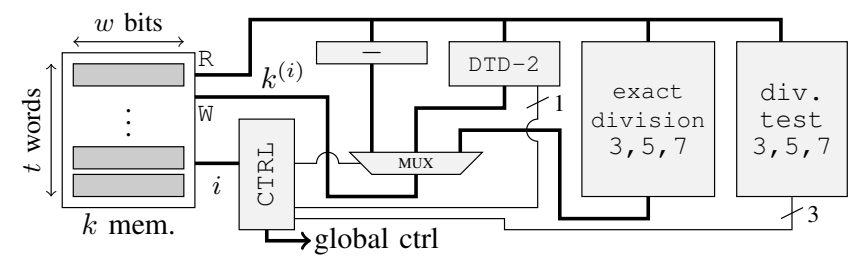

Fig. 9. Complete recoding unit architecture

described in Sec. III-C ( $w$-bit output). MUX selects which unit output should be written in the scalar memory. The global controller (CTRL) generates all control signals for units. It also provides the global control with informations on which curve-level operations have to be launched.

FPGA implementation results for the complete unsigned recoding unit are reported in Tab. $\mathrm{V}$ for $\mathcal{B}=(2,3,5,7)$ and $n=160$ (see Sec. IV-B for comparison to a ECC processor).

Our recoding unit operates significantly faster than curvelevel operations and in parallel to them. It provides on-the-fly informations on which curve-level operations to be launched without interruptions as illustrated on Fig. 10. On this figure, "CLO" denotes curve-level operations, DT denotes divisibility test, "res." their results and " $/ b_{j}$ " exact division by $b_{j}$. For $k=87$, the recoding is $87=0+3^{1} \times\left(1+2^{2} 7^{1}\right)$.

The first term $(0+3)$ recoding is performed as fast as possible while the second one $\left(1+2^{2} 7^{1}\right)$ is spread over the computations curve-level operations, without interruption. This provides us options when designing the control.

For $n=160, w=12$ and $t=14$, recoding a scalar corresponding to $2^{8} 3^{4} 5^{2} 7^{1}$ requires 292 clock cycles. This is less than one DBL operation (even for a parallel architecture). It reduces to 195 clock cycles for $w=24$ and $t=7$. The same term recoding requires 772 clocks cycles for $n=521$ and $w=12(435$ for $w=24)$.

\section{E. Validation}

Both recoding and scalar multiplication algorithms have been implemented in PARI/GP (http://pari.math.u-bordeaux.

\begin{tabular}{|c||c|c|}
\hline$w$ & $\begin{array}{c}\text { area } \\
\text { slices (FF/LUT) }\end{array}$ & $\begin{array}{c}\text { freq. } \\
\mathrm{MHz}\end{array}$ \\
\hline \hline 12 & $153(301 / 412)$ & 232 \\
\hline 24 & $323(682 / 908)$ & 202 \\
\hline \multicolumn{3}{|c|}{ TABLE V }
\end{tabular}

FPGA IMPLEMENTATION RESULTS FOR COMPLETE RECODING UNIT 


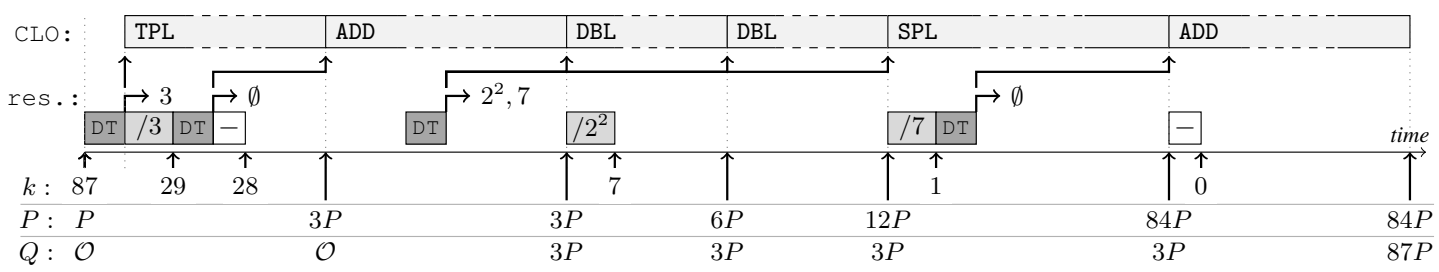

Fig. 10. MBNS recoding and scalar multiplication illustration for $k=87$

\begin{tabular}{lllll}
\hline \multicolumn{2}{c}{ unsigned version } & & & \multicolumn{2}{c}{ signed version } \\
\cline { 1 - 1 } $4:$ & $d \leftarrow 1$ & & $4:$ & $d \leftarrow \mathrm{S}(k)$ \\
$5:$ & $k \leftarrow k-1$ & & $5:$ & $k \leftarrow k-d$ \\
\hline
\end{tabular}

Fig. 11. Modifications between the unsigned and signed recoding algorithms

fr/) and SAGE (http://www.sagemath.org/) mathematical softwares (each author was in charge of one version GP or SAGE). The results of the two versions have been compared to the mathematical values and cross-checked for very large random data sets (millions of scalars for many sizes $n \in[160,520]$ bits). Functional validation of the architecture was done using some VHDL simulations on limited sets of random data and compared to the mathematical values. For performance validation, a lot of random tests and comparisons to state-ofart results have been performed (see Sec. V).

\section{Signed-Digit Optimizations}

The unsigned recoding algorithm, Fig. 3 of Sec. III-A, only performs $k \leftarrow k-1$ when $k$ is not divisible by $\mathcal{B}$ elements. As with other number systems, such as Booth recoding, $w \mathrm{NAF}$, Avizienis representations, or DBNS, using signed digits may help us to reduce the number of terms.

\section{A. Signed-Digit MBNS Recoding}

A simple modification of our MBNS recoding algorithm Fig. 3 is required to support signed digits as illustrated on Fig. 11. A selection function $\mathrm{S}$ has been introduced to select the digit $d= \pm 1$ to be used for each term $\left(d, e_{1}, e_{2}, \ldots, e_{l}\right)$ when $k$ is not divisible by $\mathcal{B}$ elements. Depending on $d$, updating the scalar requires a subtraction $(d=1$ similarly to the unsigned version) or an addition $(d=-1)$. The scalar multiplication algorithm Fig. 4 is unchanged. At curve level, digit values correspond to: a point addition when $d=1$, a point subtraction when $d=-1$ and no operation when $d=0$ (for the very first term only).

Determining $\mathrm{S}$ such that the recoding algorithm always produces the shortest list of terms is a very hard problem. We compared 4 heuristic selection functions and trade-offs between recoding performances (LT length) and implementation complexity (i.e. silicon area and recoding speed).

1) Minimum value selection function $(\mathrm{min})$ : $\mathrm{min}$ is illustrated on Fig. 12. When $k$ is not divisible, then $k-1$ and $k+1$ will be divisible by, at least, 2 (we always use $b_{1}=2$ in practice) and potentially other $b_{j}$. For each value $k-1$ and $k+1$, divisibility tests and exact division unit are used to

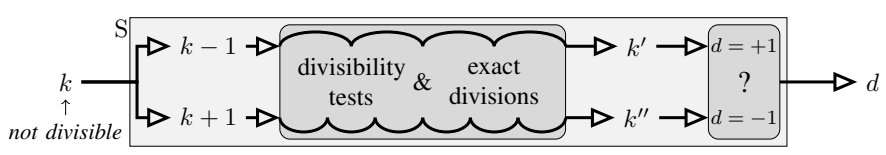

Fig. 12. Principle of the min selection function

produce $k^{\prime}$ and $k^{\prime \prime} . k^{\prime}$ (resp. $k^{\prime \prime}$ ) corresponds to $k-1$ (resp. $k+1$ ) divided as much as possible by $\mathcal{B}$ elements. S returns $d=1$ if $k^{\prime}<k^{\prime \prime}$, else it returns $d=-1$ (test $k^{\prime} \leq k^{\prime \prime}$ leads to similar performances). The min selection function only provides a local minimum for the total number of terms.

A second scalar memory ( $t$ words $\times w$ bits) has been added in the recoding unit to store both $k^{\prime}$ and $k^{\prime \prime}$. The exponents corresponding to both $k-1$ and $k+1$ have been computed and stored during the exploration. The controller is adapted such that the correct set of exponents is selected.

2) Maximum number of divisors selection function (max_nb_div): In min, half of divisibility tests and exact divisions are discarded. In max_nb_div, the number of base elements $b_{j}$ which divide $k-1$ and $k+1$ is counted. $\mathrm{S}$ returns $d$ according to the maximum number of divisors among $k-1$ and $k+1$. Only divisibility unit for $k-1$ and $k+1$ is used, not the exact division unit. max_nb_div is a cheap optimization but with low efficiency (see Sec. IV-C).

3) Approximated minimum value selection function (approx): In order to provide a cheap optimization but with higher performances, the approx selection function compares approximations of $k^{\prime}$ and $k^{\prime \prime}$ from min (instead of computing them exactly like in min). Exponents $e_{j}^{\prime}$ (resp. $e_{j}^{\prime \prime}$ ) correspond to the divisibility test results for $k-1$ (resp. $k+1)$. The approximations of $k^{\prime}$ and $k^{\prime \prime}$ are respectively defined by $\delta^{\prime}=\left\lfloor\log _{2}(k-1)\right\rfloor+1-\sum_{j=1}^{l} e_{j}^{\prime} \log _{2}\left(b_{j}\right)$ and $\delta^{\prime \prime}=\left\lfloor\log _{2}(k+1)\right\rfloor+1-\sum_{j=1}^{l} e_{j}^{\prime \prime} \log _{2}\left(b_{j}\right)$ where $\left\lfloor\log _{2}(k-1)\right\rfloor+1$ is the position of the most significant bit (MSB) of $k-1$ (idem for $k+1$ ). MSB positions can be easily detected using our divisibility test unit (during the $t$ iterations loop for each word). Approximation of weight $\prod_{j=1}^{l} b_{j}^{e^{\prime} j}$ (or with $e_{j}^{\prime \prime}$ exponents) uses the sum of multiplications by $\log _{2}\left(b_{j}\right)$ constants. Our divisibility unit returns limited exponents: $e_{j}^{\prime} \leq 1$ for $b_{j} \neq 2$ and $e_{j}^{\prime} \leq w$ for $b_{j}=2$ (see Sec. III-B). Then, there is no need for multiplications. As an example, for $\mathcal{B}=(2,3,5,7)$ $\delta^{\prime}=\operatorname{MSB}(\mathrm{k}-1)-e_{b_{1}=2}-1.5 e_{b_{2}=3}-2.25 e_{b_{3}=5}-2.75 e_{b_{4}=7}$ where $e_{b_{1}=2} \leq w$ and $e_{b_{2}=3}, e_{b_{3}=5}, e_{b_{4}=7} \leq 1$. The constants come from: $\log _{2} 3 \approx 1.59, \log _{2} 5 \approx 2.32$, and $\log _{2} 7 \approx 2.81$. 


\begin{tabular}{|c||c|c|}
\hline$w$ & $\begin{array}{c}\text { area } \\
\text { slices (FF/LUT) }\end{array}$ & $\begin{array}{c}\text { freq. } \\
\text { MHz }\end{array}$ \\
\hline \hline 12 & $173(326 / 466)$ & 232 \\
\hline 24 & $345(724 / 1005)$ & 202 \\
\hline
\end{tabular}

TABLE VI

FPGA IMPLEMENTATION RESULTS FOR SIGNED RECODING UNIT.

\begin{tabular}{|c|c|c|c|c|}
\hline \multirow[b]{2}{*}{ version } & \multirow{2}{*}{$\begin{array}{c}\text { memory } \\
\text { type }\end{array}$} & \multicolumn{2}{|l|}{ area } & \multirow{2}{*}{$\begin{array}{l}\text { freq. } \\
\mathrm{MHz}\end{array}$} \\
\hline & & slices $(\mathrm{FF} / \mathrm{LUT})$ & BRAM & \\
\hline \multirow{2}{*}{ small } & distributed & 22204 (3971/6 816) & $\overline{0}$ & $\overline{155}$ \\
\hline & BRAM & $1793(3641 / 6182)$ & 6 & 155 \\
\hline \multirow{2}{*}{ large } & distributed & $3182(4668 / 7361)$ & 0 & 142 \\
\hline & BRAM & $2427(4297 / 6981)$ & 6 & 142 \\
\hline
\end{tabular}

TABLE VII

FPGA IMPLEMENTATION RESULTS FOR A COMPLETE ECC PROCESSOR OVER $\mathbb{F}_{p}$ WITH $n=160$ BITS (FROM [25])

The approximation for both $k^{\prime}$ and $k^{\prime \prime}$, as well as their comparison, can be easily implemented using a very small circuit (see [24]).

4) 2 steps minimum value selection function (min2): It uses the time margin illustrated on Fig. 10 using a recursion limited to the next term. The first step uses min with $(k-1, k+$ $1)$ to produce $\left(k^{\prime}, k^{\prime \prime}\right)$. The second step uses min with $\left(k^{\prime}-\right.$ $\left.1, k^{\prime}+1, k^{\prime \prime}-1, k^{\prime \prime}+1\right)$ to produce $\left(\zeta^{\prime}, \zeta^{\prime \prime}, \zeta^{\prime \prime \prime}, \zeta^{\prime \prime \prime \prime \prime}\right)$. S returns $d$ according to the minimum value among $\zeta^{\prime}, \zeta^{\prime \prime}, \zeta^{\prime \prime \prime}, \zeta^{\prime \prime \prime \prime}$.

\section{B. FPGA Implementation}

Signed MBNS recoding unit has been implemented on FPGA (see end of Sec. III-B for target and tools details). Tab. VI reports corresponding results for approx selection function, $\mathcal{B}=(2,3,5,7)$ and $n=160$ bits. Compared to the unsigned version Tab. V, area overhead for signed version is very small: $13 \%$ more slices (8\% FF and 13\% LUTs). In Virtex 5 FPGAs, very small memories, such scalar ones, can be efficiently implemented using distributed RAMs in the LUTs of SLICEMs. This explains why only 25 additional flip-flops are required for the signed version while there is a 168-bit memory ( $t=14$ and $w=12$ ) for the second scalar memory. The same speed is achieved for both signed and unsigned versions (the critical path is in the exact division unit).

In order to compare our MBNS recoding unit to a complete ECC processor, Tab. VII reports two FPGA implementations of an ECC processor provided by the authors of [25] (for curves over $\mathbb{F}_{p}, n=160$ bits and Jacobian coordinates). The first one (small version) uses NAF method with one arithmetic unit per field operation, while the second (large version) uses $4 \mathrm{NAF}$ method and two arithmetic units per field operation but one modular inversion. Our MBNS signed recoding unit work at higher frequency than the ECC processor. And its area represents less than $10 \%$ (resp. $7 \%$ ) for $w=12$ compared to the complete small (resp. large) version of the ECC processor.
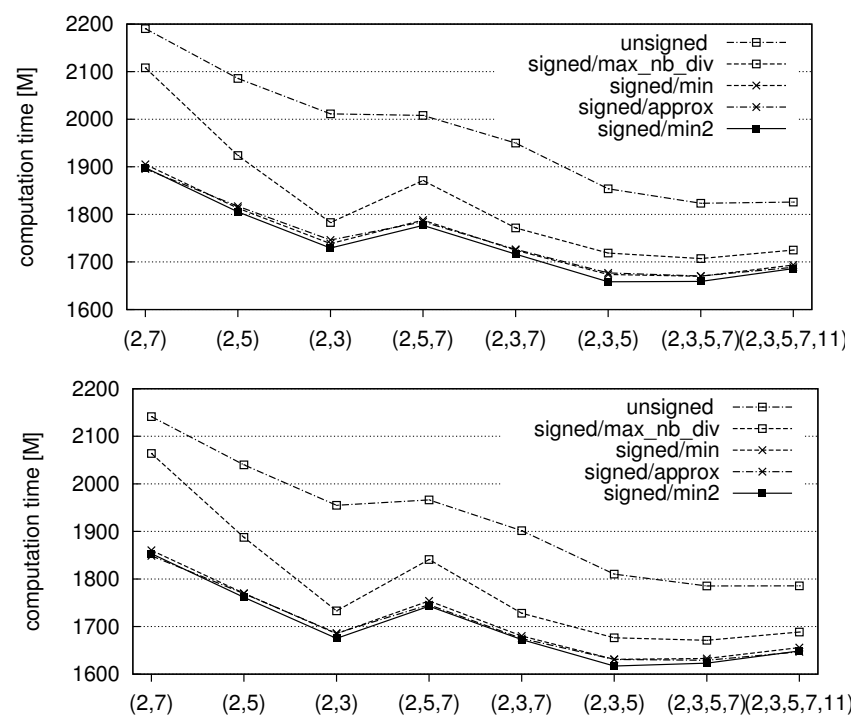

Fig. 13. Statistical performance evaluation of signed MBNS recoding and scalar multiplication (top: $a \neq-3$, bottom: $a=-3$ )

\section{Experimental Analysis}

Fig. 13 presents statistical analysis results for average computation time (in M) of 10000 scalar multiplications with 160 bit random scalars recoded using our signed MBNS algorithm for various multi-bases and the 4 selection functions presented in Sec. IV-A. Most efficient multi-bases are $\mathcal{B}=(2,3,5)$ and $\mathcal{B}=(2,3,5,7)$ with very close performances. Selection function max_nb_div is not efficient, min and approx have very close performances, and approx is the best tradeoff between performances and recoding cost (min requires longer computations and more energy).

\section{Randomized Selection Function}

Side-channel attacks exploit some correlations between secret values manipulated in the device and physical parameters measured on the device such as power consumption, electromagnetic emanations or computation timing. Refer to [26] for a complete introduction on power analysis based SCAs. Typical SCAs and counter-measures for ECC are summarized respectively in [27] and [28]. Protections against simple attacks (based on only one or a very few traces) mainly use uniformization (or atomicity) and randomization methods. Protections against differential attacks (based on statistics over many traces) mainly use randomization methods.

We experimented with a simple randomized selection function ( $r n d)$ as a protection against some SCAs. When $k$ is not divisible by $\mathcal{B}$ elements, $\mathrm{S}$ returns $d=1$ or $d=-1$ randomly. Obviously this leads to larger number of terms (and point additions) in the recoding as reported in Tab. VIII (for simplified Weierstrass curves with $a \neq-3$ and 10000 random scalars). Proposed randomization scheme allows a scalar substring to be represented using totally different recodings. This is a direct protection against some differential attacks due to the very huge number of different representations using signed digits [14, Tab. 2]. For protection against simple attacks, real 


\begin{tabular}{|c||c|c||c|c||c|}
\hline \multicolumn{1}{|c||}{$\mathcal{1}$} & \multicolumn{2}{c||}{ rnd } & \multicolumn{2}{c||}{ min } & diff. \\
\cline { 2 - 6 } & $\mathrm{M}$ & \#ADD & M & \#ADD & {$[\%]$} \\
\hline \hline$(2,3)$ & 1960.5 & 49.3 & 1738.5 & 34.0 & 12.8 \\
\hline$(2,3,5)$ & 1843.0 & 39.8 & 1673.7 & 28.0 & 10.1 \\
\hline$(2,3,5,7)$ & 1811.4 & 34.8 & 1670.0 & 24.8 & 8.5 \\
\hline$(2,3,5,7,11)$ & 1816.7 & 32.1 & 1693.5 & 22.9 & 7.3 \\
\hline
\end{tabular}

TABLE VIII

AVERAGE COMPUTATION TIME (M) AND POINT ADDITION NUMBER (\#ADD) USING THE RANDOMIZED SELECTION FUNCTION $(a \neq-3)$

robustness of our randomized selection function relies on the fact that point addition and point subtraction cannot be distinguished in traces. Indeed, protecting the sign change when using point subtraction is supposed to be simple in the circuit. But we still have to perform a more complete security evaluation at hardware level using a real attack system and to compare to other protection schemes (e.g. addition chains [29]).

\section{COMParison to State-OF-ART}

Below we compare our MBNS recoding and scalar multiplication algorithms for various multi-bases to state-of-art methods. We report results over $\mathbb{F}_{p}$ for simplified Weierstrass curves with unspecified parameter $a$ and $a=-3$, using Jacobian coordinates, similarly to most of DBNS/MBNS references.

\section{A. Costs of Curve-Level Operations}

Tab. IX reports best computation costs, given in fieldlevel operations (M, S) for various curve-level operations over $\mathbb{F}_{p}$ from literature. EFD is the excellent web site ExplicitFormulas Database http://hyperelliptic.org/EFD. We apply the typical cost assumption used in many references: $1 \mathrm{~S}=0.8 \mathrm{M}$. $\lambda$ DBL (resp. $\lambda \mathrm{TPL}$ ) denotes a sequence of $\lambda$ successive DBL (resp. TPL) operations (e.g. $k=2^{\lambda}$ or $k=3^{\lambda}$ ). We use $\lambda$ DBL or $\lambda$ TPL operations when they are faster than their equivalent sequence of DBL or TPL.

\section{B. Performance Comparisons}

Some previous scalar multiplication algorithms require additional points to speed up computations. These additional points are multiples of the initial point $P$ and stored in the cryptoprocessor during the complete scalar multiplication (2 $n$-bit registers per additional point). Most of methods assume pre-computed points represented using affine coordinates to benefit from fast mixed coordinates addition mADD. Tab. X reports costs of typical pre-computations. Costs at field-level include a conversion to affine coordinates which requires field inversions (usually $1 \mathrm{M}+1 \mathrm{~S}+2 \mathrm{I}$ per addition point). We assume $1 \mathrm{I}=15 \mathrm{M}$ for $\mathbb{F}_{p}$ inversion.

These costs can be neglected for multiple successive $[k] P$ operations with the same $P$, but it is not the case if $P$ changes before each scalar multiplication (e.g. support of various protocols/sizes, base point randomization method, ...).

Tab. XI and XII compares scalar multiplication methods from literature to our signed MBNS method using 10000 random scalars and approx selection function. In these tables, DBNS results have been computed using the PARI/GP program kindly provided by the author of [7]. This program generates a signed DBNS chain using pre-computations and where approximations are obtained by a search table.

The results presented in references [16] and [32] are based on a left-to-right MBNS scalar multiplication algorithm to benefit from mADD while the scalar is recoded using a rightto-left algorithm (this strategy prevents them from providing an on-the-fly computation). If we use a similar strategy, the computation cost reduction is estimated to $((11+5 \times 0.8)-$ $(7+4 \times 0.8))$ times the number of ADD operations. In case $\mathcal{B}=(2,3,5)$ and $n=160$, this leads to a reduction about 134M. Hence, references [16] and [32] are still faster than our method but with a much smaller difference.

\section{CONCLUSION}

In this paper, we proposed a simple multi-base recoding algorithm for ECC scalar multiplication in hardware without any pre-computations. The scalar recoding is performed on-thefly and in parallel to curve-level operations without additional latency. The proposed recoding circuit uses cheap divisibility test by multi-base elements and exact division using very small dedicated hardware units. Our MBNS recoding and scalar multiplication method is a little less competitive compared to other DBNS/MBNS methods when pre-computations or offline recoding can be used. But our method leads to more efficient solutions in embedded applications fully integrated in hardware without resources for costly recoding and limited storage. As future work, we plan to deal with more advanced recoding schemes to reduce the number of produced terms and improved randomization schemes to increase robustness against side-channel attacks.

\section{ACKNOWLEDGMENT}

We are thankful to Professor Christiane Frougny for providing us references [21], [22]. We also thank the anonymous reviewers for their valuable comments. This work has been supported in part by a $\mathrm{PhD}$ grant from Région Bretagne and Conseil Général des Côtes d'Armor (ROBUSTA project) and by the PAVOIS project (ANR 12 BS02 002 01).

\section{REFERENCES}

[1] D. Hankerson, A. Menezes, and S. Vanstone, Guide to Elliptic Curve Cryptography. Springer, 2004.

[2] H. Cohen and G. Frey, Eds., Handbook of Elliptic and Hyperelliptic Curve Cryptography. Chapman \& Hall/CRC, 2005.

[3] V. Dimitrov and T. Cooklev, "Hybrid algorithm for the computation of the matrix polynomial $I+A+\ldots+A^{N-1}$, IEEE Transactions on Circuits and Systems I: Fundamental Theory and Applications, vol. 42, no. 7, pp. 377-380, Jul. 1995.

[4] V. Dimitrov, G. Jullien, and W. Miller, "An algorithm for modular exponentiation," Information Processing Letters, vol. 66, no. 3, pp. 155159, May 1998.

[5] - "Theory and applications of the double-base number system," IEEE Trans. on Computers, vol. 48, no. 10, pp. 1098-1106, Oct. 1999.

[6] V. Dimitrov, L. Imbert, and P. K. Mishra, "Efficient and secure elliptic curve point multiplication using double-base chains," in Proc. 11th International Conference on the Theory and Application of Cryptology and Information Security (ASIACRYPT), ser. LNCS, vol. 3788 . Springer, Dec. 2005, pp. 59-78. 


\begin{tabular}{|c|c|c|c|c|c|c|c|c|}
\hline \multirow[b]{2}{*}{ curves } & \multirow[b]{2}{*}{ references } & \multicolumn{7}{|c|}{ curve-level operations } \\
\hline & & ADD & mADD & DBL & TPL & QPL & SPL & EPL \\
\hline \multirow{3}{*}{$a \neq-3$} & EFD & $11 M+5 S$ & $7 \mathrm{M}+4 \mathrm{~S}$ & $1 M+8 S$ & $5 \mathrm{M}+10 \mathrm{~S}$ & n. a. & n. a. & n. a. \\
\hline & {$[30]$} & n. a. & n. a. & $1 M+8 S$ & $5 \mathrm{M}+10 \mathrm{~S}$ & $7 \mathrm{M}+16 \mathrm{~S}$ & $15 \mathrm{M}+24 \mathrm{~S}$ & $17 \mathrm{M}+30 \mathrm{~S}$ \\
\hline & [31] & $11 M+5 S$ & $7 \mathrm{M}+4 \mathrm{~S}$ & $2 \mathrm{M}+8 \mathrm{~S}$ & $6 \mathrm{M}+11 \mathrm{~S}$ & $9 \mathrm{M}+15 \mathrm{~S}$ & $13 \mathrm{M}+18 \mathrm{~S}$ & n. a. \\
\hline \multirow{3}{*}{$a=-3$} & EFD & $11 M+5 S$ & $7 \mathrm{M}+4 \mathrm{~S}$ & $3 M+5 S$ & $7 \mathrm{M}+7 \mathrm{~S}$ & n. a. & n. a. & n. a. \\
\hline & {$[32]$} & $11 M+5 S$ & $7 \mathrm{M}+4 \mathrm{~S}$ & $3 \mathrm{M}+5 \mathrm{~S}$ & $7 \mathrm{M}+7 \mathrm{~S}$ & $11 \mathrm{M}+11 \mathrm{~S}$ & $18 \mathrm{M}+11 \mathrm{~S}$ & $28 \mathrm{M}+15 \mathrm{~S}$ \\
\hline & {$[16],[31]$} & $11 M+5 S$ & $7 \mathrm{M}+4 \mathrm{~S}$ & $3 \mathrm{M}+5 \mathrm{~S}$ & $7 \mathrm{M}+8 \mathrm{~S}$ & $10 \mathrm{M}+12 \mathrm{~S}$ & $14 \mathrm{M}+15 \mathrm{~S}$ & n. a. \\
\hline curves & references & \multicolumn{3}{|c|}{$\lambda \mathrm{DBL}$} & \multicolumn{4}{|c|}{$\lambda \mathrm{TPL}$} \\
\hline$a \neq-3$ & {$[6],[11],[33]$} & \multicolumn{3}{|c|}{$4 \lambda \mathrm{M}+(4 \lambda+2) \mathrm{S}$} & \multicolumn{4}{|c|}{$(11 \lambda-1) \mathrm{M}+(4 \lambda+2) \mathrm{S}$} \\
\hline curves & references & \multicolumn{7}{|c|}{$\lambda \mathrm{TPL} / \lambda^{\prime} \mathrm{DBL}$} \\
\hline$a \neq-3$ & [6], [11] & \multicolumn{7}{|c|}{$\left(11 \lambda+4 \lambda^{\prime}-1\right) \mathrm{M}+\left(4 \lambda+4 \lambda^{\prime}+3\right) \mathrm{S}$} \\
\hline
\end{tabular}

TABLE IX

COSTS OF CURVE-LEVEL OPERATIONS FROM LITERATURE AND CURVES OVER $\mathbb{F}_{p}$

\begin{tabular}{|c|c|c|c|c|c|}
\hline \multirow{2}{*}{ pre-computations } & \multirow{2}{*}{ methods } & \multicolumn{4}{|c|}{ computation time } \\
\hline & & & & $a \neq-3$ & $a=-3$ \\
\hline \multirow{2}{*}{$3 P$} & $3 \mathrm{NAF}$ & $1 \mathrm{DB}$ & $+1 \mathrm{mADD}$ & $49.4 \mathrm{M}$ & $49.0 \mathrm{M}$ \\
\hline & DBNS & & 1TPL & $44.8 \mathrm{M}$ & $44.4 \mathrm{M}$ \\
\hline \multirow{2}{*}{$3 P, 5 P, 7 P$} & 4NAF & $2 \mathrm{DB}$ & $+3 \mathrm{mADD}$ & $140.8 \mathrm{M}$ & $140.0 \mathrm{M}$ \\
\hline & DBNS & $1 \mathrm{TPL}+1 \mathrm{DB}$ & $+2 \mathrm{mADD}$ & $136.2 \mathrm{M}$ & $135.4 \mathrm{M}$ \\
\hline
\end{tabular}

TABLE $X$

TYPICAL COSTS OF PRE-COMPUTATIONS FOR ADDITIONAL POINTS

\begin{tabular}{|c|c|c|c|c|c|}
\hline \multirow[b]{2}{*}{ references } & \multirow[b]{2}{*}{ methods } & \multirow[b]{2}{*}{ performances } & \multicolumn{2}{|c|}{ pre-computations } & \multirow[b]{2}{*}{ recoding } \\
\hline & & & storage & operations & \\
\hline & double-and-add & $1985.3 \mathrm{M}$ & $\emptyset$ & $\emptyset$ & $\emptyset$ \\
\hline & NAF & $1723.0 \mathrm{M}$ & $\emptyset$ & $\emptyset$ & on-the-fly \& very cheap \\
\hline & 3NAF & $1583.7 \mathrm{M}$ & 1 point & $49.4 \mathrm{M}$ & on-the-fly \& very cheap \\
\hline & $4 \mathrm{NAF}$ & $1499.1 \mathrm{M}$ & 3 points & $140.8 \mathrm{M}$ & on-the-fly \& very cheap \\
\hline [6] & DBNS & $1863.0 \mathrm{M}$ & $\emptyset$ & $\emptyset$ & off-line \& costly \\
\hline [11] & DBNS & $1722.3 \mathrm{M}$ & $\emptyset$ & $\emptyset$ & off-line \& costly \\
\hline [9] & DBNS & $1558.4 \mathrm{M}$ & 7 points & $>150 \mathrm{M}$ & off-line \& costly \\
\hline [7] & DBNS & $1615.3 \mathrm{M}$ & $\emptyset$ & $\emptyset$ & off-line \& costly \\
\hline & $(2,3) \mathrm{MBNS}$ & $1746.2 \mathrm{M}$ & $\emptyset$ & $\emptyset$ & on-the-fly \& small \\
\hline this work & $(2,3,5) \mathrm{MBNS}$ & $1679.9 \mathrm{M}$ & $\emptyset$ & $\emptyset$ & on-the-fly \& small \\
\hline & $(2,3,5,7) \mathrm{MBNS}$ & $1670.4 \mathrm{M}$ & $\emptyset$ & $\emptyset$ & on-the-fly \& small \\
\hline
\end{tabular}

TABLE XI

COMPARISON OF SCALAR MULTIPLICATION METHODS (CURVES OVER $\mathbb{F}_{p}$ WITH $a \neq-3$ AND $n=160$ )

[7] C. Doche and L. Imbert, "Extended double-base number system with applications to elliptic curve cryptography," in Proc. 7th International Conference on Cryptology (INDOCRYPT), ser. LNCS, vol. 4329. Kolkata, India: Springer, Dec. 2006, pp. 335-348.

[8] R. Barua, S. K. Pandey, and R. Pankaj, "Efficient window-based scalar multiplication on elliptic curves using double-base number system," in Proc. 8th International Conference on Progress in Cryptology (INDOCRYPT), ser. LNCS, vol. 4859. Springer, Dec. 2007, pp. 351-360.

[9] D. J. Bernstein, P. Birkner, T. Lange, and C. Peters, "Optimizing double-base elliptic-curve single-scalar multiplication," in Proc. 8th International Conference on Progress in Cryptology (INDOCRYPT), ser. LNCS, vol. 4859. Springer, Dec. 2007, pp. 167-182.

[10] C. Doche and L. Habsieger, "A tree-based approach for computing double-base chains," in Proc. 13th Australasian Conference on Informa- tion Security and Privacy, ser. LNCS, vol. 5107, Jul. 2008, pp. 433-446.

[11] V. Dimitrov, L. Imbert, and P. K. Mishra, "The double-base number system and its application to elliptic curve cryptography," Mathematics of Computation, vol. 77, no. 262, pp. 1075-1104, Apr. 2008.

[12] S. Singha, A. Ghosh, and A. Sinha, "A new architecture for FPGA based implementation of conversion of binary to double base number system (DBNS) using parallel search technique," ACM SIGARCH Computer Architecture News, vol. 39, no. 5, pp. 12-18, Dec. 2011.

[13] T. Chabrier, D. Pamula, and A. Tisserand, "Hardware implementation of DBNS recoding for ECC processor," in Asilomar Conference on Signals, Systems and Computers. IEEE, Nov. 2010, pp. 1129-1133.

[14] P. K. Mishra and V. Dimitrov, "Efficient quintuple formulas for elliptic curves and efficient scalar multiplication using multibase number representation," in Proc. 10th International Conference on Information 


\begin{tabular}{|c|c|c|c|c|c|}
\hline \multirow[b]{2}{*}{ references } & \multirow[b]{2}{*}{ methods } & \multirow[b]{2}{*}{ performances } & \multicolumn{2}{|c|}{ pre-computations } & \multirow[b]{2}{*}{ recoding } \\
\hline & & & storage & operations & \\
\hline & double-and-add & $1922.0 \mathrm{M}$ & $\emptyset$ & $\emptyset$ & $\emptyset$ \\
\hline & NAF & $1659.7 \mathrm{M}$ & $\emptyset$ & $\emptyset$ & on-the-fly \& very cheap \\
\hline & $3 \mathrm{NAF}$ & $1520.2 \mathrm{M}$ & 1 point & $49.0 \mathrm{M}$ & on-the-fly \& very cheap \\
\hline & 4NAF & $1436.1 \mathrm{M}$ & 3 points & $140.0 \mathrm{M}$ & on-the-fly \& very cheap \\
\hline [7] & DBNS & $1563.2 \mathrm{M}$ & $\emptyset$ & $\emptyset$ & off-line \& costly \\
\hline [9] & DBNS & $1504.3 \mathrm{M}$ & 7 points & $>150 \mathrm{M}$ & off-line \& costly \\
\hline \multirow{5}{*}{ [14] } & \multirow{5}{*}{$(2,3,5) \mathrm{MBNS}$} & $1645.4 \mathrm{M}$ & $\emptyset$ & $\emptyset$ & off-line $\&$ costly \\
\hline & & $1606.4 \mathrm{M}$ & 1 point & $\approx 45 \mathrm{M}$ & off-line \& costly \\
\hline & & $1566.4 \mathrm{M}$ & 3 points & $\approx 150 \mathrm{M}$ & off-line \& costly \\
\hline & & $1552.3 \mathrm{M}$ & 7 points & $>150 \mathrm{M}$ & off-line \& costly \\
\hline & & $1486.4 \mathrm{M}$ & 5 points & $>150 \mathrm{M}$ & off-line \& costly \\
\hline \multirow{9}{*}[32]{} & $(2,3) \mathrm{NAF}$ & $1514.0 \mathrm{M}$ & $\emptyset$ & $\emptyset$ & small \\
\hline & $(2,3,5) \mathrm{NAF}$ & $1490.0 \mathrm{M}$ & $\emptyset$ & $\emptyset$ & small \\
\hline & $(2,3,5,7) \mathrm{NAF}$ & $1491.0 \mathrm{M}$ & $\emptyset$ & $\emptyset$ & small \\
\hline & $(2,3) \mathrm{NAF}_{3}$ & $1460.0 \mathrm{M}$ & 1 point & $\approx 45 \mathrm{M}$ & small \\
\hline & $(2,3,5) \mathrm{NAF}_{3}$ & $1444.0 \mathrm{M}$ & 1 point & $\approx 45 \mathrm{M}$ & small \\
\hline & $(2,3,5,7) \mathrm{NAF}_{3}$ & $1449.0 \mathrm{M}$ & 1 point & $\approx 45 \mathrm{M}$ & small \\
\hline & $(2,3) \mathrm{NAF}_{4}$ & $1384.0 \mathrm{M}$ & 3 points & $>150 \mathrm{M}$ & small \\
\hline & $(2,3,5) \mathrm{NAF}_{4}$ & $1383.0 \mathrm{M}$ & 3 points & $>150 \mathrm{M}$ & small \\
\hline & $(2,3,5,7) \mathrm{NAF}_{4}$ & $1394.0 \mathrm{M}$ & 3 points & $>150 \mathrm{M}$ & small \\
\hline \multirow{2}{*}{ [16] } & $(2,3,5) \mathrm{NAF}$ & $1460.0 \mathrm{M}$ & $\emptyset$ & $\emptyset$ & costly \\
\hline & $(2,3,5) \mathrm{NAF}$ & $1426.0 \mathrm{M}$ & 6 points & $>150 \mathrm{M}$ & costly \\
\hline \multirow{3}{*}{ this work } & $(2,3) \mathrm{MBNS}$ & $1686.2 \mathrm{M}$ & $\emptyset$ & $\emptyset$ & on-the-fly \& small \\
\hline & $(2,3,5) \mathrm{MBNS}$ & $1631.0 \mathrm{M}$ & $\emptyset$ & $\emptyset$ & on-the-fly \& small \\
\hline & $(2,3,5,7) \mathrm{MBNS}$ & $1629.3 \mathrm{M}$ & $\emptyset$ & $\emptyset$ & on-the-fly \& small \\
\hline
\end{tabular}

TABLE XII

COMPARISON OF SCALAR MULTIPLICATION METHODS (CURVES OVER $\mathbb{F}_{p}$ WITH $a=-3$ AND $n=160$ )

Security (ISC), ser. LNCS, vol. 4779, Oct. 2007, pp. 390-406.

[15] P. Longa, "Accelerating the scalar multiplication on elliptic curve cryptosystems over prime fields," Master's thesis, Univ. Ottawa, 2007.

[16] P. Longa and C. Gebotys, "Fast multibase methods and other several optimizations for elliptic curve scalar multiplication," in Proc. Public Key Cryptography (PKC), ser. LNCS, vol. 5443, 2009, pp. 443-462.

[17] G. N. Purohit and A. S. Rawat, "Fast scalar multiplication in ECC using the multi base number system," International Journal of Computer Science Issues, vol. 8, no. 1, pp. 131-137, May 2011.

[18] X. Yin, T. Yang, and J. Ning, "Optimized approach for computing multibase chains," in Proc. 7th International Conference on Computational Intelligence and Security (CIS). IEEE, Dec. 2011, pp. 964-968.

[19] J. Adikari, V. S. Dimitrov, and L. Imbert, "Hybrid binary-ternary number system for elliptic curve cryptosystems," IEEE Transactions on Computers, vol. 60, no. 2, pp. 254-265, Feb. 2011.

[20] G. Purohit, A. S. Rawat, and M. Kumar, "Elliptic curve point multiplication using MBNR and point halving," International Journal of Advanced Networking and Applications, vol. 3, no. 5, pp. 1329-1337, 2012.

[21] B. Pascal, Euvres complètes. Librarie Lefèvre, 1819, vol. 5, ch. De Numeribus Multiplicibus, pp. 117-128.

[22] J. Sakarovitch, Elements of Automata Theory. Cambridge, 2009, ch. Prologue: M. Pascal's Division Machine, pp. 1-6.

[23] T. Jebelean, "An algorithm for exact division," Journal of Symbolic Computation, vol. 15, no. 2, pp. 169-180, Feb. 1993.

[24] N. Boullis and A. Tisserand, "Some optimizations of hardware multiplication by constant matrices," IEEE Transactions on Computers, vol. 54 no. 10, pp. 1271-1282, Oct. 2005.

[25] A. Byrne, E. Popovici, and W. Marnane, "Versatile processor for $\operatorname{gf}\left(p^{m}\right)$ arithmetic for use in cryptographic applications," IET Computers \& Digital Techniques, vol. 2, no. 4, pp. 253-264, Jul. 2008.
[26] S. Mangard, E. Oswald, and T. Popp, Power Analysis Attacks: Revealing the Secrets of Smart Cards. Springer, 2007.

[27] E. Oswald, Advances in Elliptic Curve Cryptography, ser. London Mathematical Society Lecture Note Series. Cambridge University Press, Apr. 2005, vol. 317, ch. Side Channel Analysis, pp. 69-86.

[28] M. Joye, Advances in Elliptic Curve Cryptography, ser. London Mathematical Society Lecture Note. Cambridge University Press, Apr. 2005, vol. 317, ch. Defenses Against Side-Channel Analysis, pp. 87-100.

[29] A. Byrne, N. Meloni, A. Tisserand, E. M. Popovici, and W. P. Marnane, "Comparison of simple power analysis attack resistant algorithms for an elliptic curve cryptosystem," Journal of Computers, vol. 2, no. 10, pp. $52-62,2007$.

[30] P. Giorgi, L. Imbert, and T. Izard, "Optimizing elliptic curve scalar multiplication for small scalars," in Proc. Mathematics for Signal and Information Processing, vol. 7444. SPIE, Sep. 2009, pp. 74 440N:1-10.

[31] P. Longa and C. Gebotys, "Setting speed records with the (fractional) multibase non-adjacent form method for efficient elliptic curve scalar multiplication," Cryptology ePrint Archive, Tech. Rep. 118, 2008.

[32] P. Longa and A. Miri, "New multibase non-adjacent form scalar multiplication and its application to elliptic curve cryptosystems," IACR Eprint, Tech. Rep. 52, 2008.

[33] K. Itoh, M. Takenaka, N. Torii, S. Temma, and Y. Kurihara, "Fast implementation of public-key cryptography on a DSP TMS320C6201," in Proc. Cryptographic Hardware and Embedded Systems (CHES), ser. LNCS, vol. 1717. Springer, Aug. 1999, pp. 61-72. 